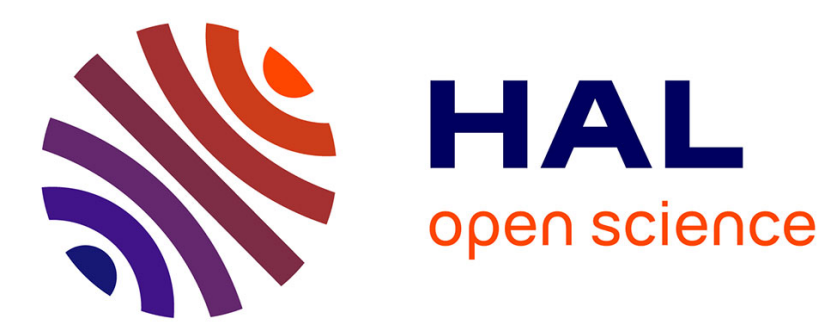

\title{
Multi-Water-Bag Model And Method Of Moments For The Vlasov Equation
}

Anaïs Crestetto, Philippe Helluy

\section{To cite this version:}

Anaïs Crestetto, Philippe Helluy. Multi-Water-Bag Model And Method Of Moments For The Vlasov Equation. Finite Volumes for Complex Applications VI, Jun 2011, Prague, Czech Republic. pp.293301, 10.1007/978-3-642-20671-9_31 . hal-00641991

\section{HAL Id: hal-00641991 https://hal.science/hal-00641991}

Submitted on 17 Nov 2011

HAL is a multi-disciplinary open access archive for the deposit and dissemination of scientific research documents, whether they are published or not. The documents may come from teaching and research institutions in France or abroad, or from public or private research centers.
L'archive ouverte pluridisciplinaire HAL, est destinée au dépôt et à la diffusion de documents scientifiques de niveau recherche, publiés ou non, émanant des établissements d'enseignement et de recherche français ou étrangers, des laboratoires publics ou privés. 


\title{
Multi-Water-Bag Model And Method Of Moments For The Vlasov Equation
}

\author{
Anaïs Crestetto * $\quad$ Philippe Helluy ${ }^{\dagger}$
}

\begin{abstract}
The kinetic Vlasov-Poisson model is very expensive to solve numerically. It can be approximated by a multi-water-bag model in order to reduce the complexity. This model amounts to solve a set of Burgers equations, which can be done easily by finite volume methods. However, the solution is naturally multivalued (filamentation). The multivalued solution can be computed by a moment method. We present here several numerical experiments.
\end{abstract}

Keywords: Vlasov-Poisson, water-bag approximation, Burgers equation, multivalued solution MSC2010: 35Q83, 44A60, 65M08

\section{Introduction}

Kinetic equations are used in several domains, such as plasma physics or bubble flows in gases or liquids. The distribution function depends on space and time but also on an additional velocity variable. Computations are thus very expensive. It is of great interest to reduce the complexity of the resolution by using fluid methods.

We consider a plasma containing ions of positive charge and electrons of negative charge. Ions are much heavier than electrons so that we can neglect their displacement and assume that their density $n_{0}$ is constant. The electrons move following the system of Vlasov-Poisson in a periodic domain in $\mathbf{x}$ :

$$
\begin{aligned}
\partial_{t} f(\mathbf{x}, \mathbf{v}, t) & +\mathbf{v} \cdot \nabla_{\mathbf{x}} f(\mathbf{x}, \mathbf{v}, t)+\frac{q}{m} \mathbf{E}(\mathbf{x}, t) \cdot \nabla_{\mathbf{v}} f(\mathbf{x}, \mathbf{v}, t)=0 \\
\operatorname{div} \mathbf{E}(\mathbf{x}, t) & =\rho(\mathbf{x}, t)=\frac{q}{m}\left(\int f(\mathbf{x}, \mathbf{v}, t) d \mathbf{v}-n_{0}\right) \\
\int \mathbf{E}(\mathbf{x}, t) d \mathbf{x} & =0 \\
f(\mathbf{x}, \mathbf{v}, 0) & =f_{0}(\mathbf{x}, \mathbf{v}) \approx n_{0}
\end{aligned}
$$

The unknowns are the distribution function of electrons $f$, and the electric field $\mathbf{E}$. The electric field depends on space and time $\mathbf{x}, t$, while the distribution function depends also on an additional velocity variable $\mathbf{v}$. The charge and the mass of one electron are noted $q<0$ and $m>0$ respectively. Without loss of generality, we can take $\frac{q}{m}=-1$ and $n_{0}=1$. In one dimension of space, this system becomes:

$$
\begin{aligned}
\partial_{t} f(x, v, t) & +v \partial_{x} f(x, v, t)-E(x, t) \partial_{v} f(x, v, t)=0, \\
\partial_{x} E(x, t) & =\rho(x, t)=1-\int f(x, v, t) d v \\
\int E(x, t) d x & =0 \\
f(x, v, 0) & =f_{0}(x, v) \approx 1 .
\end{aligned}
$$

\footnotetext{
*Calvi, INRIA Nancy - Grand Est \& IRMA, Université de Strasbourg

${ }^{\dagger}$ IRMA, Université de Strasbourg
} 
The distribution function $f$ is initially a perturbation of the equilibrium $n_{0}$. After simple calculations, Equation (6) can also be written

$$
\partial_{t} E(x, t)=\int v f(x, v, t) d v-\int v f(0, v, t) d v .
$$

In higher dimensions, Equation (6) would be replaced by a Poisson equation, assuming that $E=-\nabla \Phi$, where $\Phi$ is the electric potential.

The solution can be stable (for example in the case of Landau damping). But since there is no dissipation, the solution can become unstable and filamentation can appear.

In order to reduce the complexity of our model, we propose to approximate the kinetic equation by fluid models. We consider two possibilities:

- the multi-water-bag model,

- the method of moments.

Then we propose numerical approximations of these models by simple finite volume schemes. The numerical results will be compared to those obtained with a full resolution of the kinetic model by the popular Particle-In-Cell (PIC) method (described for example in [3]).

\section{Multi-water-bag model}

The multi-water-bag model, detailed in [2], generalizes the water-bag model (see for example [1]). It consists of replacing $f$ by a piecewise constant approximation in the velocity variable. Each piece is called a "water-bag". It is possible to compute only the boundaries of the water-bags.

\subsection{Presentation of the model}

Let $N$ be an integer, $v_{j}^{+}(x, t)$ and $v_{j}^{-}(x, t)$ velocities, $A_{j}$ constants, $j=1, \ldots, N$, such that we can write:

$$
f(x, v, t)=\sum_{j=1}^{N} A_{j}\left(H\left(v_{j}^{+}(x, t)-v\right)-H\left(v_{j}^{-}(x, t)-v\right)\right),
$$

where $H$ is the Heaviside function: $H(u)=\left\{\begin{array}{l}0 \text { if } u<0, \\ 1 \text { if } u>0 .\end{array}\right.$

Injecting this expression in the Vlasov equation, and assuming that there is no two $v_{j}^{ \pm}(x, t)$ equal, we obtain the following system:

$$
\begin{aligned}
\partial_{t} v_{j}^{ \pm} & +v_{j}^{ \pm} \partial_{x} v_{j}^{ \pm}+E=0, \forall j=1, \ldots, N \\
\partial_{t} E & =-\sum_{j=1}^{N} A_{j}\left(\frac{v_{j}^{+^{2}}(x, 0)-v_{j}^{-2}(x, 0)}{2}-\frac{v_{j}^{+^{2}}(x, t)-v_{j}^{-{ }^{2}}(x, t)}{2}\right) .
\end{aligned}
$$

The velocities follow a Burgers equation with a source term. Instead of evolving the distribution function $f$, we evolve these velocities. The natural solution can become multivalued (filamentation). The weak entropy solution is only an approximation in this context, when shocks appear.

\section{$2.2 \quad$ Numerical scheme}

We discretize our domain: $x_{i}=x_{0}+i \Delta x$, with $\Delta x$ being the spacial step, and consider a time step $\Delta t$ such that $t^{n}=n \Delta t$. We evolve each velocity independently by using, for example, the Godunov scheme:

$$
v_{j, i}^{ \pm, n+1}=v_{j, i}^{ \pm, n}-\frac{\Delta t}{\Delta x}\left(\frac{\left(v_{j, i+\frac{1}{2}}^{ \pm, \star}\right)^{2}}{2}-\frac{\left(v_{j, i-\frac{1}{2}}^{ \pm, \star}\right)^{2}}{2}\right)-\Delta t E_{i}^{n},
$$


where $v_{j, i}^{ \pm, n} \simeq v_{j}^{ \pm}\left(x_{i}, t^{n}\right)$ and $E_{i}^{n} \simeq E\left(x_{i}, t^{n}\right)$. We compute the $v_{j}^{ \pm, \star}$ with an exact Riemann solver.

We compute the electric field with the following scheme:

$$
E_{i}^{n+1}=E_{i}^{n}-\Delta t \sum_{j=1}^{N} A_{j}\left(\frac{\left(v_{j, i}^{+, 0}\right)^{2}-\left(v_{j, i}^{-, 0}\right)^{2}}{2}-\frac{\left(v_{j, i}^{+, n}\right)^{2}-\left(v_{j, i}^{-, n}\right)^{2}}{2}\right) .
$$

In higher dimension, this step should be replaced by the numerical resolution of a Poisson equation.

\section{$2.3 \quad$ Remarks}

Before the shock, the weak solution and the multivalued solution coincide. After the shock, the natural solution is multivalued, filaments or branches appear, and the weak solution becomes discontinuous. In Section 4, we compare numerically the two kinds of solutions. It is also possible to compute several branches by the method of moments [4].

\section{Method of moments}

The method of moments, presented in $[5,6]$, consists in taking the first moments of the equation that we have to solve in order to reduce the number of variables. The system is closed by assuming that the distribution function is made of water-bags.

\subsection{Presentation of the method}

Definition 1: The moment $M_{k}$ of order $k$ of $f$ is defined by:

$$
M_{k}(x, t)=\int_{-\infty}^{+\infty} v^{k} f(x, v, t) d v
$$

Taking the $2 N$ first moments of the Vlasov equation:

$$
\int v^{k} \partial_{t} f(x, v, t) d v+\int v^{k+1} \partial_{x} f(x, v, t) d v-E(x, t) \int v^{k} \partial_{v} f(x, v, t) d v=0,
$$

we obtain the following system of moments, coupled with the equation for the electric field:

$$
\begin{aligned}
\partial_{t} M_{0}+\partial_{x} M_{1} & =0 \\
\partial_{t} M_{k}+\partial_{x} M_{k+1} & +k E M_{k-1}=0, \text { for } k=1, \ldots, 2 N-1, \\
\partial_{t} E(x, t) & =M_{1}(x, t)-M_{1}(0, t) .
\end{aligned}
$$

We now have a system of $2 N+1$ equations, in which the velocity no longer appears. There are $2 N+2$ unknowns: the moments $M_{k}$ for $k=0, \ldots, 2 N$ and $E$. We have to close this system by finding an expression for $M_{2 N}$.

\subsection{Closure relation}

We represent $f$ by water-bags for closing the system:

$$
f(x, v, t)=\sum_{j=1}^{N} A_{j}\left(H\left(v_{j}^{+}(x, t)-v\right)-H\left(v_{j}^{-}(x, t)-v\right)\right) .
$$

We obtain:

$$
M_{k}(x, t)=\sum_{j=1}^{N} A_{j} \frac{v_{j}^{+^{k+1}}(x, t)-v_{j}^{-k+1}(x, t)}{k+1}, \forall k=0, \ldots, 2 N,
$$

and thus have an expression of $M_{2 N}$, assuming that we know the $v_{j}^{ \pm}$. 


\subsection{Numerical scheme}

At time $t^{n}$, we know the $2 N$ first moments of $f$ and the electric field. We solve the system:

$$
M_{k}(x, t)=\sum_{j=1}^{N} A_{j} \frac{v_{j}^{+^{k+1}}(x, t)-v_{j}^{-k+1}(x, t)}{k+1}, \forall k=0, \ldots, 2 N-1,
$$

to obtain $v_{j}^{ \pm}$, at time $t^{n}$. In general, the system may have several solutions. Uniqueness can be recovered through an entropy argument [4].

To solve this system, we can use the Newton method. But we have no rigorous result of convergence.

When the problem is such that $f$ can be written:

$$
f(x, v, t)=\sum_{j=1}^{2 N}(-1)^{j-1} H\left(a_{j}^{+}(x, t)-v\right),
$$

where $-\infty<a_{2 N} \leq \cdots \leq a_{1}<+\infty$, we can use the algorithm described in [4, 7], which solves rigorously such a system. It is then possible to catch numerically the multivalued solutions.

For approximating the system of moments, we use a natural kinetic scheme. Formally, we write :

$$
\partial_{t} f(x, v, t)+v^{+} \partial_{x} f(x, v, t)+v^{-} \partial_{x} f(x, v, t)-E(x, t) \partial_{v} f(x, v, t)=0,
$$

where $v^{+}=\max (0, v)$ and $v^{-}=\min (0, v)$. Denoting $\Delta x$ as the space step, $\Delta t$ as the time step, and $f_{i}^{n}$ as the approximation of $f\left(x_{i}, v, t^{n}\right)$, we use the following upwind discretization for the Vlasov equation:

$$
\frac{f_{i}^{n+1}-f_{i}^{n}}{\Delta t}+\frac{1}{\Delta x}\left(v^{+}\left(f_{i}^{n}-f_{i-1}^{n}\right)+v^{-}\left(f_{i+1}^{n}-f_{i}^{n}\right)\right)-E_{i}^{n} \partial_{v} f_{i}^{n}=0 .
$$

We multiply it by $v^{k}$ and integrate it in $v$ :

$$
\begin{aligned}
& \frac{1}{\Delta t} \int v^{k}\left(f_{i}^{n+1}-f_{i}^{n}\right) d v \\
+ & \frac{1}{\Delta x} \int\left(v^{+^{k+1}}\left(f_{i}^{n}-f_{i-1}^{n}\right)+v^{-k+1}\left(f_{i+1}^{n}-f_{i}^{n}\right)\right) d v \\
- & E_{i}^{n} \int v^{k} \partial_{v} f_{i}^{n} d v=0 .
\end{aligned}
$$

We obtain a finite volume scheme:

$$
\frac{M_{k, i}^{n+1}-M_{k, i}^{n}}{\Delta t}+\frac{1}{\Delta x}\left(F\left(M_{k, i}^{n}, M_{k, i+1}^{n}\right)-F\left(M_{k, i-1}^{n}, M_{k, i}^{n}\right)\right)-k E_{i}^{n} M_{k-1, i}^{n}=0 .
$$

The scheme for the electric field is:

$$
\frac{E_{i}^{n+1}-E_{i}^{n}}{\Delta t}=M_{1, i}^{n}-M_{1,0}^{n}
$$

\section{Numerical results}

We validate our models on classical test cases: Landau damping and two stream instability, and obtain good decrease rates for the electric potential energy, when $N$ is big enough. An example for a Landau damping test case is given in Figure 1, with $N=5$.

We are now interested in solutions that can initially exactly be depicted by the multi-waterbag model, and that are unstable. We compare the three methods in Figure 2 , for $N=1$ :

- the method of moments with an approximation by water-bags,

- the multi-water-bag model with the scheme of Godunov,

- the Particle-In-Cell (PIC) method, considered as the reference.

Before the shock, the two fluid methods describe precisely the solution. After the shock, they describe the main part of the solution, but cannot catch the filaments. More test cases will be presented at the conference, with higher $N$. 


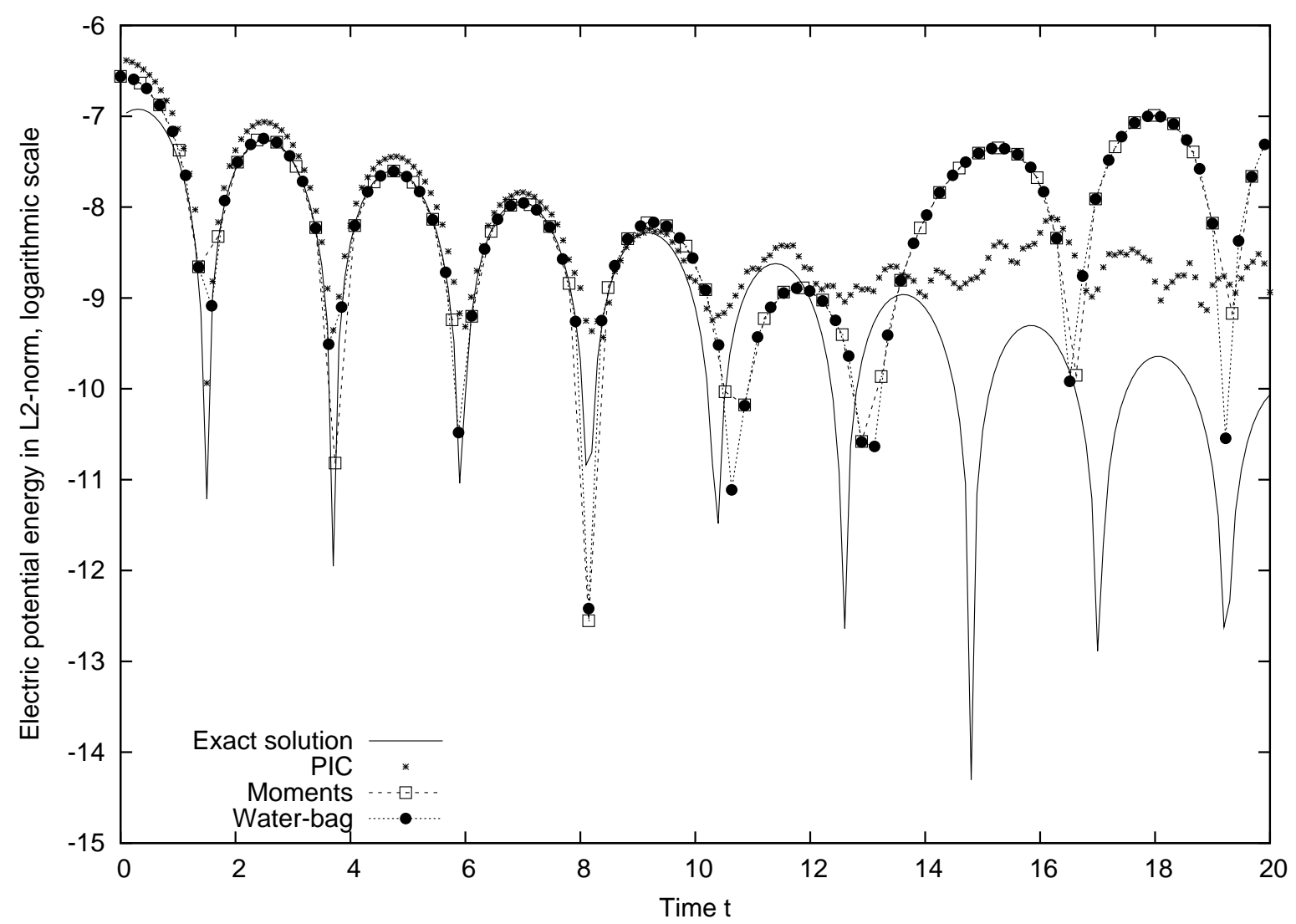

Figure 1: Landau damping. Multi-water-bag model, method of moments and PIC method compared to the exact solution.

\section{References}

[1] Bertrand, P., Feix, M. R.: Non linear electron plasma oscillation: the water bag model. Phys. Lett. 28A, 68-69 (1968)

[2] Besse, N., Berthelin, F., Brenier, Y., Bertrand, P.: The multi-water-bag equations for collisionless kinetic modeling. Kinetic and Related Models 2, 39-80 (2009)

[3] Birdsall, C. K. and Langdon, A. B.: Plasma Physics via Computer Simulation. Institute of Physics (1991)

[4] Brenier, Y. and Corrias, L.: A kinetic formulation for multi-branch entropy solutions of scalar conservation laws. Annales de l'Institut Henri Poincaré. Analyse Non Linéaire 15, 169-190 (1998)

[5] Desjardins, O., Fox, R. O., Villedieu, P.: A quadrature-based moment method for dilute fluid-particle flows. Journal of Computational Physics 227, 2514-2539 (2008)

[6] Fox, R. O., Laurent, F., Massot, M.: Numerical simulation of spray coalescence in an Eulerian framework: direct quadrature method of moments and multi-fluid method. Journal of Computational Physics 227, 3058-3088 (2008)

[7] Gosse, L. and Runborg, O.: Resolution of the finite Markov moment problem. Comptes Rendus Mathématique. Académie des Sciences. Paris 12, 775-780 (2005) 

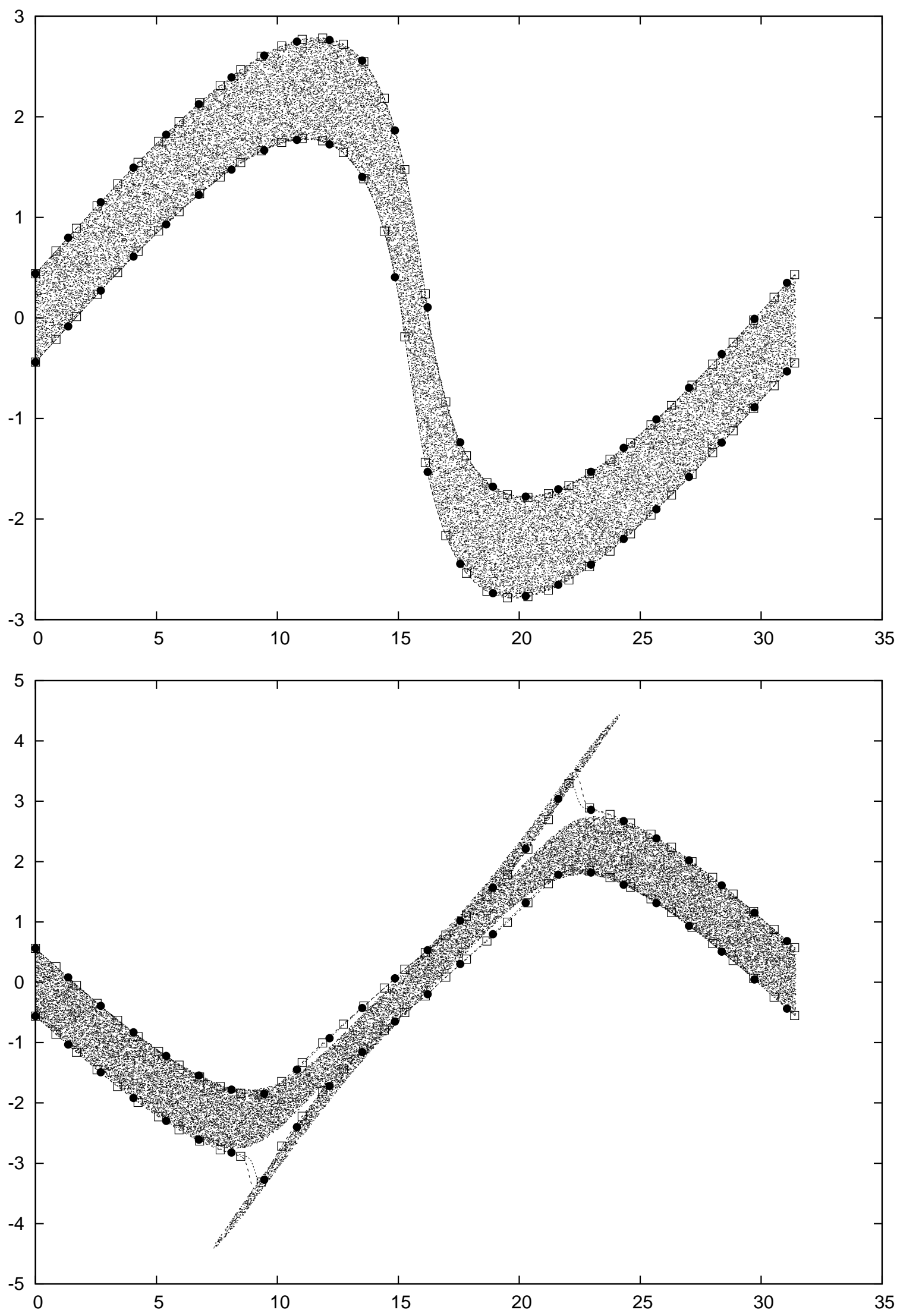

Figure 2: Test case for $N=1$ in the phase space at times $T=2$ and $T=5$. Multi-water-bag model (black circles), method of moments (empty squares) and PIC method (dots). 